\title{
Comparative growth analysis of five first year establishment poplar clones (Populus sp.) grown under a short-rotation intensive culture system
}

\author{
F. Mau and I. Impens
}

Laboratory of Planty Ecology, Department of Biology, University of Antwerp, Universiteitsplein 1 , 2610 Wilrijk, Belgium

\section{Introduction}

Interest in fast growing poplar clones for maximum production in short-rotation intensive culture is obvious. Clones from Aigeiros and Tacamahaca sections are well suited for short-rotation intensive culture because of their rapid juvenile growth.

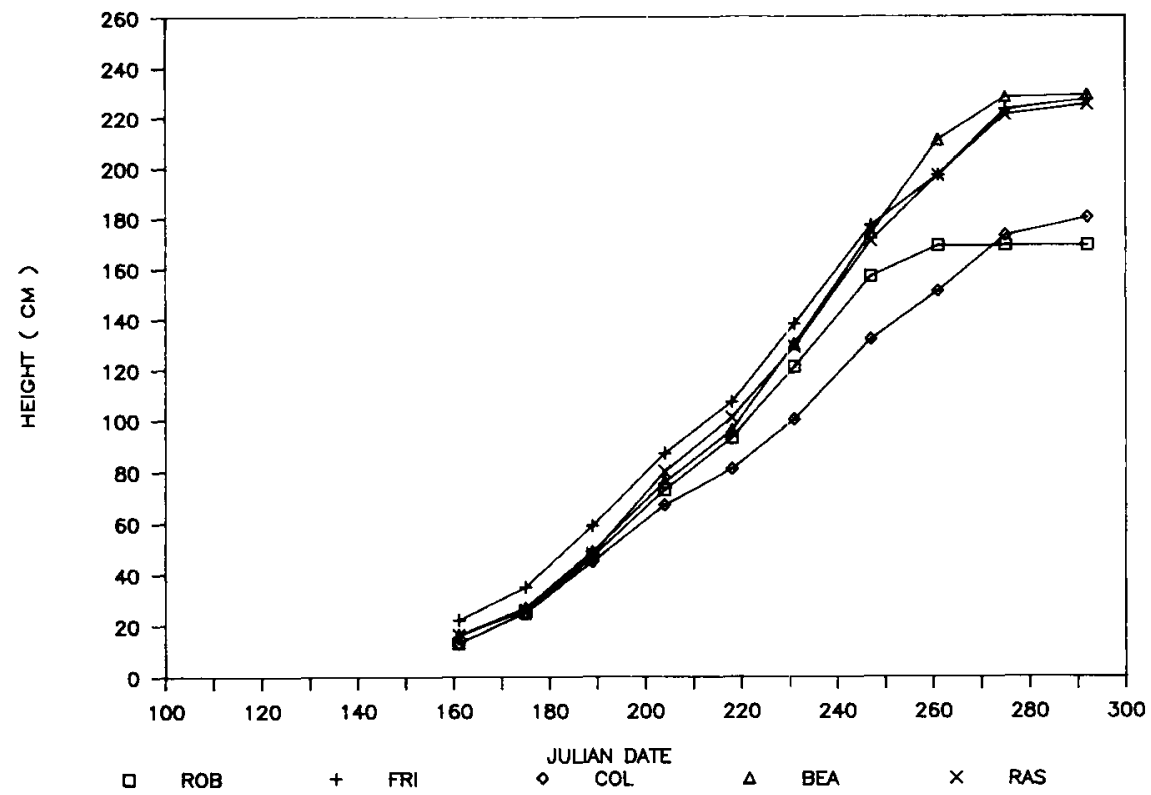

Fig. 1. Mean tree height $(\mathrm{cm})$ of 8 model trees for 5 clones $(\mathrm{ROB}=$ Robusta, $\mathrm{FRI}=$ Fritzi Pauley, $\mathrm{COL}=$ Columbia River, BEA = Beaupré, RAS = Raspalje) increasing with date (Julian date $1=$ Jan. 1, 1987). 


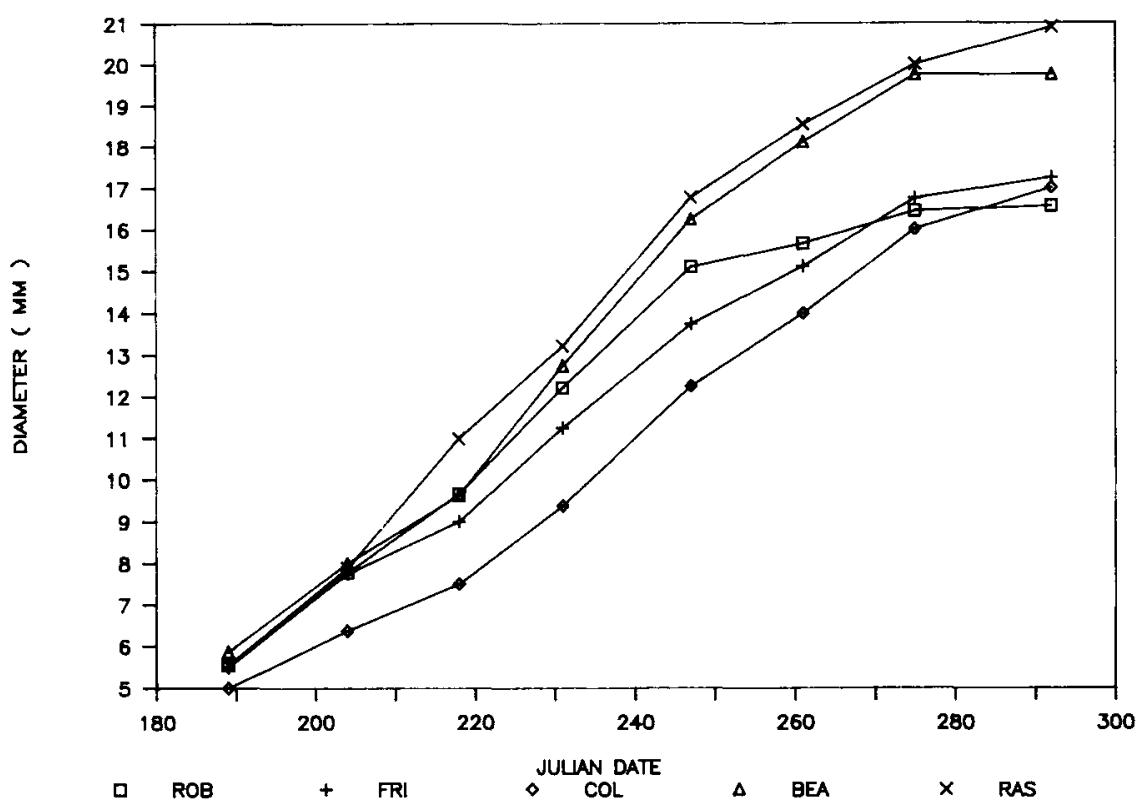

Fig. 2. Mean stem diameter (mm) of 8 model trees for 5 clones (see legend to Fig. 1) increasing with date (Julian date 1 = Jan. 1, 1987).

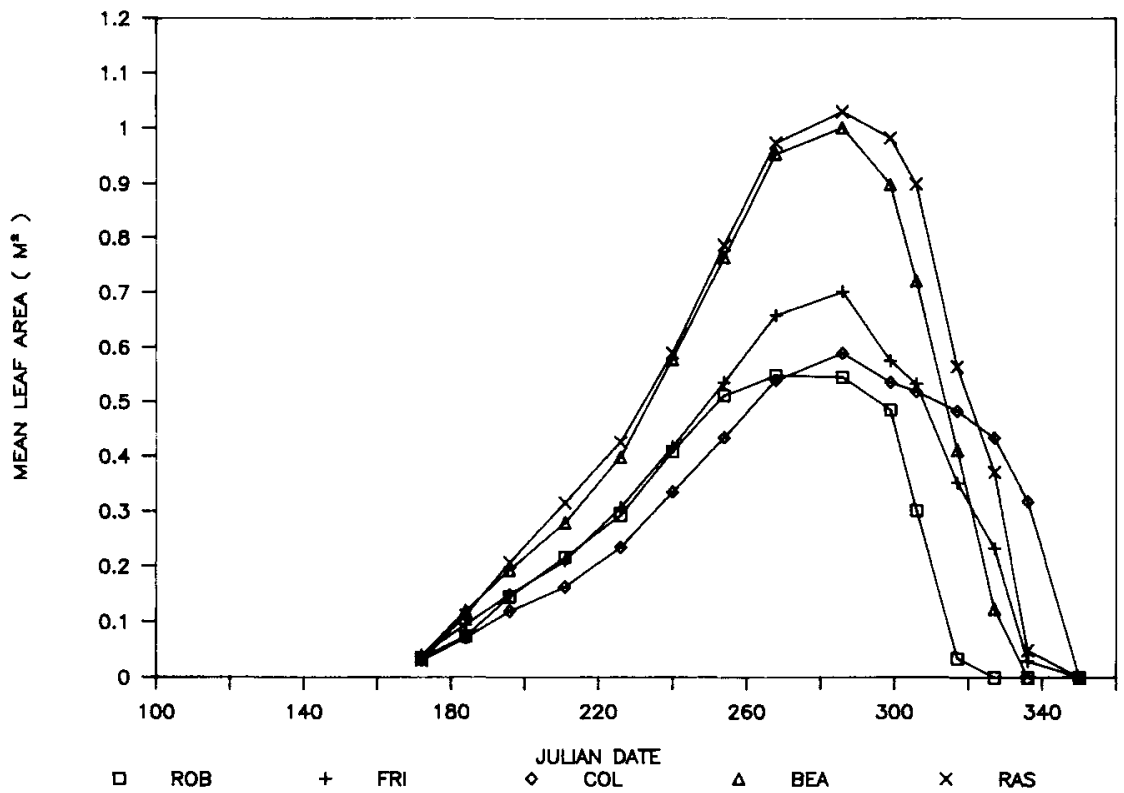

Fig. 3. Mean leaf area development/tree according to date (Julian date 1 = Jan. 1 1987) of 8 model trees for 5 clones (see legend to Fig. 1). 
'Fast starter' clones are most suited for a 1-3 yr old coppice stand rotation according to Zsuffa (1979). Superior hybrid growth was indicated as a factor responsible for the good performance of hybrid poplar in comparison with mere species clones (Cain and Ormrod, 1984).

\section{Materials and Methods}

Five poplar clones: $P . \times$ euramericana $\mathrm{cv}$. Robusta; P. trichocarpa cv. Fritzi Pauley; P. trichocarpa cv. Columbia River; $P$. trichocarpa $x$ $P$. deltoides cv. Beaupré; $P$. trichocarpa $\times P$. deltoides cv. Raspalje; were grown from cuttings planted in a $0.80 \times 0.80 \mathrm{~m}$ pattern at Afsnee $\left(51^{\circ} 02^{\prime} \mathrm{N}, 3^{\circ} 39^{\prime} \mathrm{E}\right.$; Belgium) in 1987 . The trees were well irrigated, fertilized and the plots kept free of weeds.
Table I. Mean leaf area duration of 8 model trees in days for 5 clones $(\mathrm{ROB}=$ Robusta, $\mathrm{FRI}=$ Fritzi Pauley, $\mathrm{COL}=$ Columbia River , $\mathrm{BEA}=$ Beaupré, $\mathrm{RAS}=$ Raspalje) .

\begin{tabular}{lc}
\hline Clone & $\begin{array}{c}\text { Leaf area duration } \\
(x \pm S D \text { in } d)\end{array}$ \\
\hline ROB & $77 \pm 12$ \\
FRI & $99 \pm 15$ \\
COL & $95 \pm 22$ \\
BEA & $134 \pm 34$ \\
RAS & $148 \pm 20$ \\
\hline
\end{tabular}

I = not significantly different at level 0.05 (Spjotvoll-Stoline $T$-test).

Primary parameters, such as basal diameter and height, were measured every 2 wk for each plot consisting of 81 trees. Height was measured to an accuracy of $0.5 \mathrm{~cm}$ by means of a measuring rod and diameter was determined on each stem with a vernier caliper $22 \mathrm{~cm}$ above

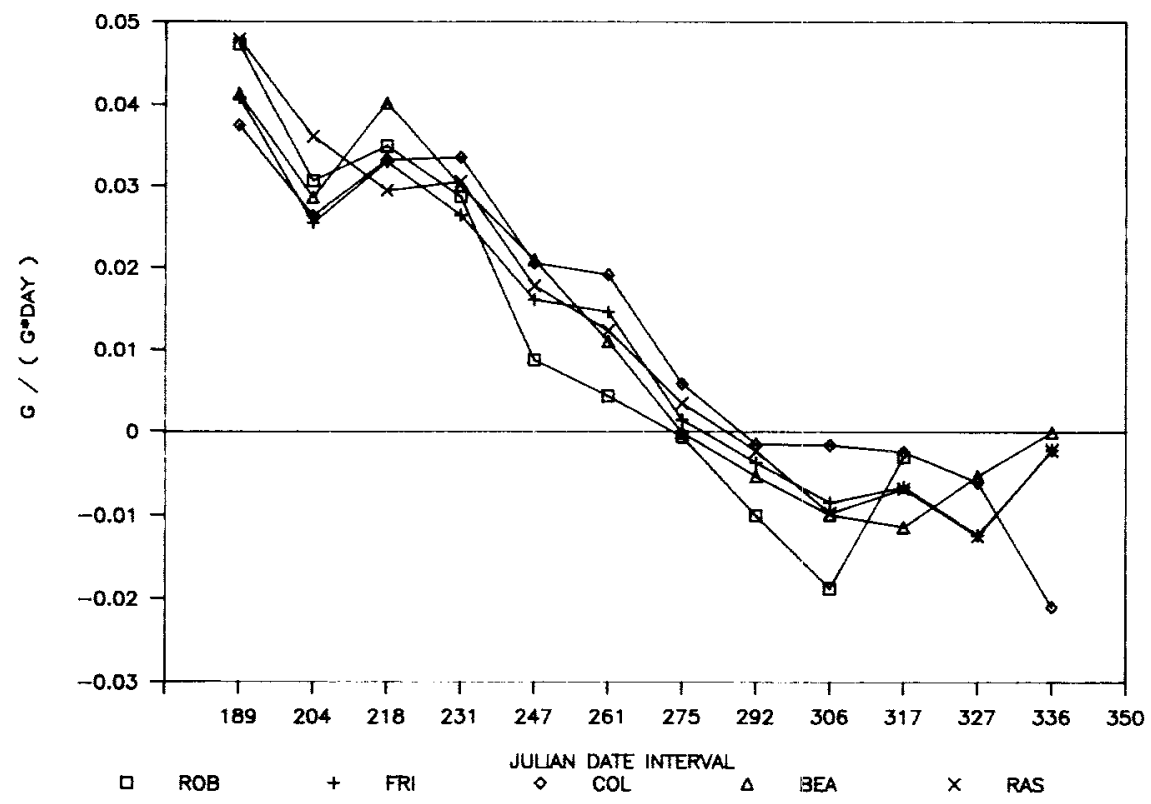

Fig. 4. Mean relative growth rate change with date (Julian date $1=$ Jan. 1,1987 ) of 8 model trees for 5 clones (see legend to Fig. 1) in terms of grams aboveground dry weight (g) change per day and per gram aboveground dry weight present at the beginning of each time interval $( \pm 14$ days). 


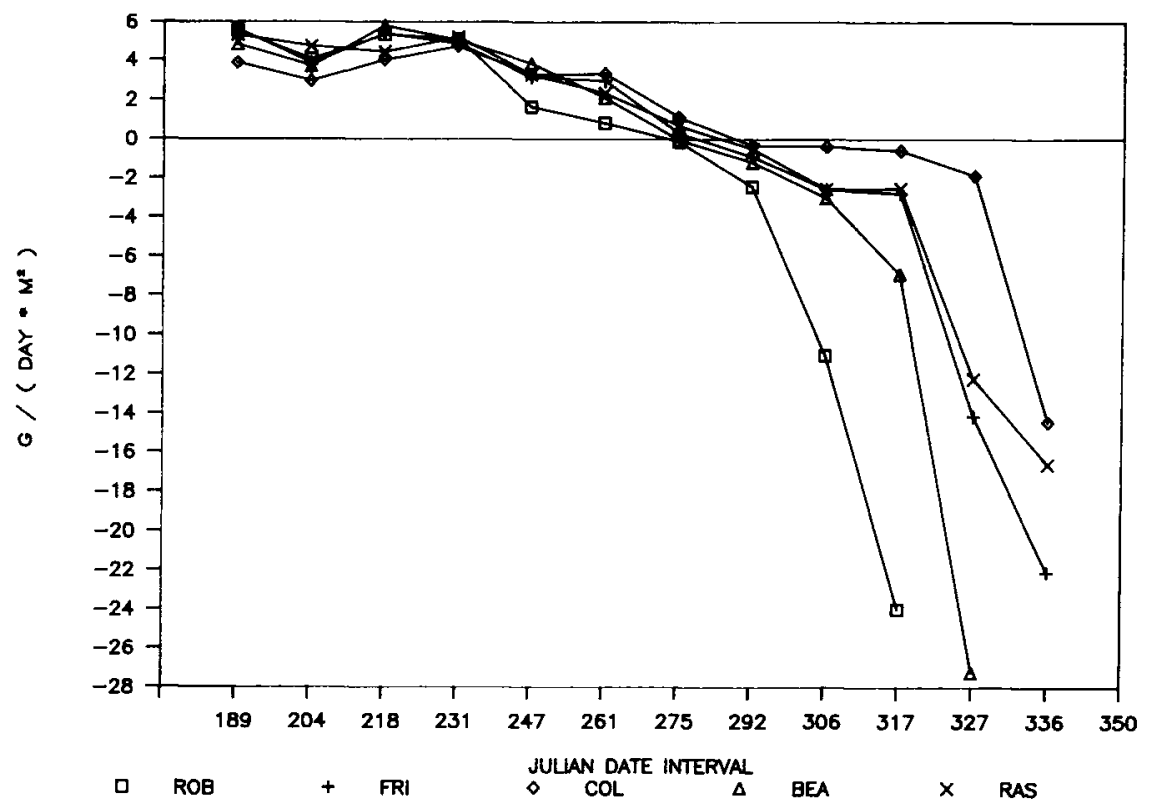

Fig. 5. Mean net assimilation rate evolution with date (Julian date $1=\mathrm{Jan} .1,1987$ ) of 8 model trees for 5 clones (see legend to Fig. 1) in terms of grams dry weight $(g)$ per day and per square meter $\left(\mathrm{m}^{2}\right)$ leat area present at the beginning of each time interval $( \pm 14 \mathrm{~d})$.

ground level. Other parameters, such as numbers of leaves, height of leaf implantation and leaf orientation, were monitored on 8 model trees of each clone. Leaf development, leaf demography and leaf area distribution were studied in relation to aboveground leaf and wood biomass productions. Leaf area was calculated from allometric relations between leaf area and leaf dimensions. Seasonal growth was also analyzed in terms of relative growth rate (RGR), net assimilation rate (NAR) and leaf area ratio $(L A R)$ with $R G R=N A R \times L A R$. At the end of the growing season, biomass production was estimated by destructive sampling and weight-surface (leaves) or weight-volume (stems) relationships.

\section{Results and Discussion}

Stems of Raspalje, Fritzi Pauley, Beaupré (about $227 \mathrm{~cm}$ ) were higher than those of
Robusta and Columbia River with respectively 169 and $180 \mathrm{~cm}$. Robusta ended height growth early around Julian date 261 (Fig. 1).

Stem diameter was the largest with Beaupré and Raspalje (20 and $22 \mathrm{~mm}$ ), while the diameter growth of Robusta had already culminated around Julian date 247 (Fig. 2).

Maximum leaf area was reached around Julian date 286, Raspalje and Beaupré having considerably higher leaf areas (about $1 \mathrm{~m}^{2}$ ) than the other clones. Robusta had the shortest period of leaf retention, in comparison with Columbia River for which $50 \%$ of the leaf area was retained until 3 wk later (Fig. 3). Leaf area duration data (Table I) emphasize the large differences among clones. 


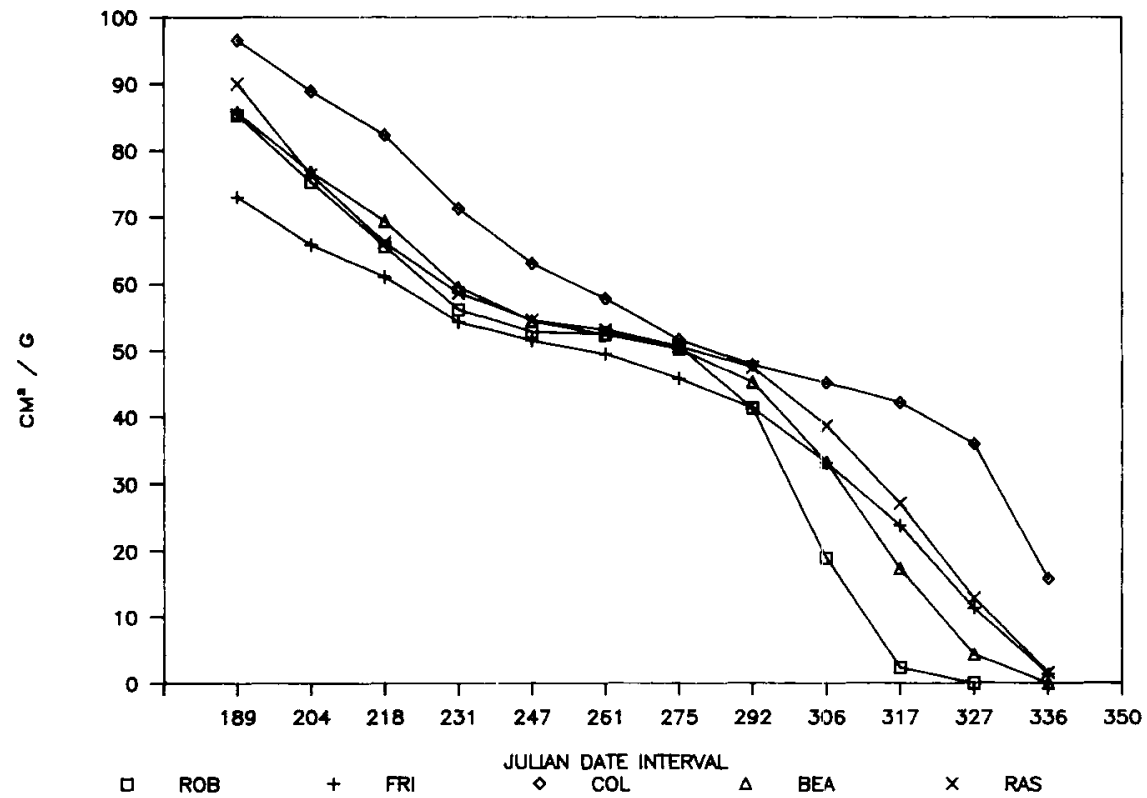

Fig. 6. Mean leaf area ratio change with date (Julian date $1=$ Jan. 1, 1987) of 8 model trees for 5 clones (see legend to Fig. 1) in terms of leaf area $\left(\mathrm{cm}^{2}\right)$ per gram aboveground dry weight.

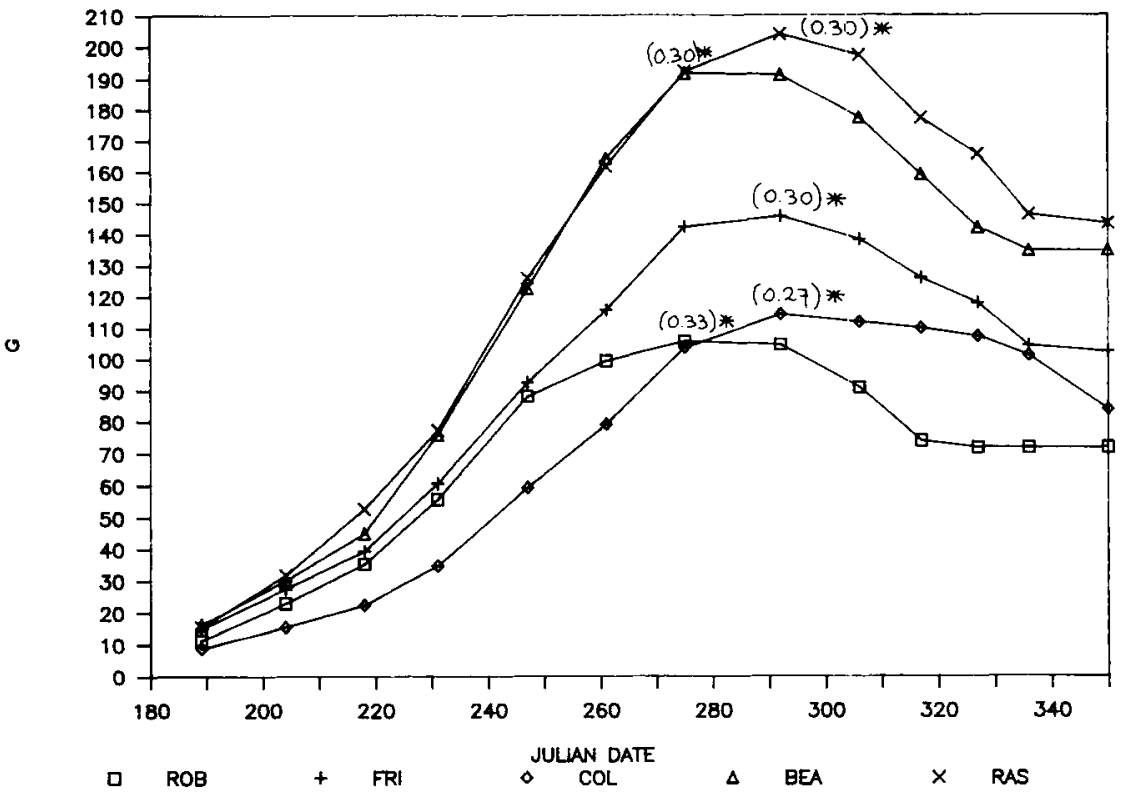

Fig. 7. Mean total aboveground biomass change with date (Julian date $1=$ Jan. 1,1987 ) of 8 model trees for 5 clones (see legend to Fig. 1) in terms of grams dry weight $(\mathrm{g})$. 
The RGR decreased from the beginning of the season, as was also shown by Farmer (1980), and became negative (leaf fall) at the end (Fig. 4). The NAR was also diminishing giving negative values at the end of the growing season and showing a large difference between Robusta and Columbia River at that time (Fig. 5). The $\angle A R$ decreased throughout the season; Columbia River always had the highest ratio (Fig. 6).

Aboveground dry biomass production varied by a factor of 2 between the most productive clone (Raspalje) and the least productive one (Robusta). The ratio between leaf dry biomass and total aboveground dry biomass, however, was about the same for all clones at maximal aboveground standing crop (Fig. 7; $\mathrm{ROB}=0.33$, $\mathrm{FRI}=0.30, \mathrm{COL}=0.27, \mathrm{BEA}=0.30$, RAS $=0.30$ ).

The best performing hybrids were Raspalje and Beaupré. In contrast, Robusta had the lowest values for most of the growth parameters evaluated.

\section{Acknowledgments}

This study was supported by the I.W.O.N.L. (Brussels, Belgium) file no. 860370 .

\section{References}

Cain N.P. \& Ormrod D.P. (1984) Hybrid vigour as indicated by early growth characteristics of Populus deltoides, P. nigra and P. $x$ euramericana. Can. J. Bot. 62, 1-8

Farmer R.E. Jr. (1980) Comparative analysis of 1 st-year growth in six deciduous tree species. Can. J. For. Res. 10, 35-41

Zsuffa L. (1979) The features and prospects of poplar breeding in Ontario. In: Poplar Research, Management and Utilization in Canada. (Fayle D.C.F., Zsuffa L. \& Anderson H.W., eds.), Ont. Min. Nat. Resources For. Res. Inf. Paper \#102, 1-1 to 1-6 